\title{
SISTEMA DE VOTAÇÃO ELETRÔNICA PARA A COMISSÃO INTERNA DE PREVENÇÃO DE ACIDENTES
}

\section{ELECTRONIC VOTING SYSTEM FOR CIPA}

\author{
Renan Galvão Alves ${ }^{1}$ \\ ${ }^{1}$ Fundação Educacional de Andradina, Faculdades Integradas Stella Maris \\ E-mail: renan gal@hotmail.com
}

\begin{abstract}
RESUMO - Este trabalho teve como objetivo desenvolver uma ferramenta capaz de agilizar o processo de votação e apuração de votos para a CIPA (Comissão Interna de Prevenção de Acidentes), bem como relatórios que disponibilizam informações importantes sobre esta eleição, ao garantir o sigilo e a ética durante todo o processo. Para tanto, foi utilizada a linguagem de programação Object Pascal com a plataforma de desenvolvimento Embarcadero Delphi 2002, gerenciador de banco de dados IBExpert, com banco de dados Firebird versão 1.5, Erwin 7.3, utilizado para fazer a modelagem de dados lógico e físico deste projeto e Microsoft Visio 2013 para desenhar os diagramas. Constatou-se que tal programa, por meio de ferramentas computacionais, atende as principais necessidades das empresas, sobretudo no caso da CIPA, por ser um ambiente de desenvolvimento eficaz e de baixo custo, além de agilizar o processo de votação e apuração dos votos.

Palavras-chaves: CIPA; eleição; votação.
\end{abstract}

ABSTRACT - The objective of this research was to develop a tool capable of
streamlining the voting process and counting of votes for the CIPA (Internal
Commission for the Prevention of Accidents), as well as reports that provide
important information about this election, while ensuring confidentiality and
ethics during the entire process. For that, the programming language Object
Pascal was used with the development platform Embarcadero Delphi 2002,
database manager IBExpert, with database manager IBExpert, with database
Firebird version 1.5 , Erwin 7.3, used to make the logical and physical data modeling of this project and Microsoft Visio 2013 to draw the diagrams. It was found that such a program, by means of computational tools, meets the main needs of companies, especially in the case of CIPA, because it is an effective and low-cost development environment, in addition to speeding up the process of voting and counting votes.

Keywords: CIPA; election; to vote.

\section{INTRODUÇÃO}

O presente projeto de desenvolvimento é um recorte do Trabalho de Conclusão de Curso do autor deste artigo, defendido no ano de 2015, na cidade de Andradina-SP. O referido teve como propósito criar um sistema de urna eletrônica, o qual será detalhado ao longo deste texto.

De acordo com Pacheco e Tait (2000), torna-se difícil que as empresas dentre as suas rotinas deixem de fazer o uso da tecnologia para desempenhar suas respectivas funções organizacionais, por meio do uso dos 
computadores que se tornam uma ferramenta indispensável para realizar as tarefas de rotina e prestação de serviços aos seus clientes. Além de contar com uma infraestrutura adequada, os softwares que serão utilizados também deverão passar por uma criteriosa metodologia de análise para que depois de implantado, possam atender as demandas da instituição.

Diante da evolução tecnológica, as empresas de produção e prestação de serviços sofreram grandes mudanças em suas rotinas, tais como a automatização nos serviços bancários, comunicação via chat on-line, compra e venda de mercadorias via internet, caixa de supermercados, controle de estoque, cursos à distância, entre outros. Quando aplicada corretamente, a tecnologia gera agilidade no processo, evitando desperdícios, otimizando recursos, aumentando assim os ganhos e tornando-se assim algo indispensável em suas rotinas (CÔRTES, 2008).

Nesse sentido, juntamente com a necessidade dos interessados, ao fazer um recorte do tema dos softwares, neste artigo o foco estará atrelado ao desenvolvimento de um software para a votação eletrônica, de tal forma que oportunize considerar a ambivalência entre: alto custo $X$ falta de recursos financeiros, indisponibilidade de manutenção $X$ alta complexidade do software, entre outros, criando assim uma falta de entendimento entre usuário, desenvolvedor, necessidades da empresa e produto desenvolvido.

De acordo com Schauren (2016), o modelo de urna eletrônica utilizado após as eleições de 1994 ajudou a diminuir a quantidade de problemas no voto por cédulas de papel, tais como problemas na caligrafia do eleitor, nome incompleto ou simplesmente inseriam o apelido do candidato. Tudo isso poderia acarretar, dependendo do caso, anulação ou destinação do voto a outro que não o escolhido, ocorriam também manipulações nos resultados na contagem das cédulas ou mesmo nos mapas de votação, sendo que poderia ser alterado os resultados finais, mesmo após a contagem das cédulas. O meio eletrônico além de resolver estes problemas, por consequência, trouxe agilidade na apuração e na divulgação dos resultados.

Atualmente, a maioria das empresas ainda realizam o processo de votação por meio de cédulas de papel, sendo que nesse processo o eleitor recebe uma cédula de votação que contém o nome de todos os candidatos, assinala o escolhido e o deposita em urna lacrada durante o período de votação. Os funcionários assinam também uma lista com seus respectivos nomes para que não haja duplicidade na votação. Ao final, a urna é aberta perante a Comissão Eleitoral, representantes dos empregados, do empregador e a quem quiser acompanhar a apuração. De acordo com a Norma Regulamentadora (NR5) do Ministério do Trabalho (SEGURANÇA E MEDICINA DO TRABALHO, 2007, p.54):

O processo eleitoral observará as seguintes condições: a. publicação e divulgação de edital, em locais de fácil acesso e visualização, no prazo mínimo de 45 (quarenta e cinco) dias antes do término do mandato em curso; b. inscrição e eleição individual, sendo que o período mínimo para inscrição será de quinze dias; c. liberdade de inscrição para todos os empregados do estabelecimento, independentemente de setores ou locais de trabalho, com fornecimento de comprovante; d. garantia de emprego para todos os inscritos até a eleição; e. realização da eleição no prazo mínimo de 30 (trinta) dias antes do término do mandato da CIPA, quando houver; $f$. realização de eleição em dia normal de trabalho, respeitando os horários de turnos e em horário que possibilite a participação da maioria dos empregados; g. voto secreto; h. apuração dos votos, em horário normal de trabalho, com acompanhamento de representante do empregador e dos empregados, em número a ser definido pela comissão eleitoral; i. faculdade de eleição por meios eletrônicos; j. 
guarda, pelo empregador, de todos os documentos relativos à eleição, por um período mínimo de cinco anos.

Embora, o item "i" pontue que é facultado a eleição ocorrer por meios eletrônicos, o presente desenvolvimento justifica-se diante da possibilidade de informatizar esse processo, garantindo maior agilidade e lisura.

\section{MÉTODO}

No desenvolvimento foi utilizada a linguagem de programação Object Pascal com a plataforma de desenvolvimento Embarcadero Delphi XE7 2002, gerenciador de banco de dados IBExpert 2012.5.19.1, com banco de dados Firebird versão 1.5, Erwin 7.3, utilizado para fazer a modelagem de dados lógico e físico deste projeto e Microsoft Visio 2013 para desenhar os diagramas.

Conforme Cristóvão (2002), o Delphi, lançado em 1995, trouxe uma nova perspectiva na utilização do Pascal, pois a linguagem que é utilizada no Delphi é uma versão adaptada desta, não sendo assim uma nova linguagem que os programadores tiveram que aprender e se familiarizarem. Em 1995 ocorria o mesmo cenário de quando foi lançado o Pascal, sendo utilizado o Visual $\mathbf{C}++$ que dispunha de certa complexidade para trabalhar com recursos de baixo nível no Windows, existia também o Basic que logo mais foi nomeado de Visual Basic, que com novas ferramentas facilitou a vida dos programadores, embora alguns problemas existentes no Basic continuassem se fazendo presentes. Com o lançamento do Delphi, a Borland tornou-se líder de mercado e referência no segmento, indiscutivelmente a ferramenta continua sendo a melhor, entre as existentes no mercado. Vale lembrar também que tanto o Delphi como o Visual Basic se aperfeiçoaram e disputam a liderança como principais linguagens de programação para o Windows.
Um teste piloto foi realizado na empresa Ambulatório Médico de Especialidades - AME, localizada em Andradina-SP, para garantir maior confiabilidade a esse processo. Na época, a empresa contava com um sistema que gerenciava os setores de Recursos Humanos, Departamento Pessoal e a Segurança e Medicina do Trabalho, utilizando o módulo "RM" da empresa "TOTVS".

Nesse sistema, já existia o módulo para realizar a votação para eleição dos candidatos da CIPA, porém este contava com um sistema online, ou seja, os colaboradores da empresa que não dispunham de computadores com acesso à internet em seus respectivos setores ficariam desprivilegiados por não contribuírem com os seus votos, ao ocasionar um baixo volume de participação, o que, por consequência, poderia anular a eleição. Também deve ser considerado que a ativação deste módulo, no sistema, faz parte de um pacote à parte, o qual geraria grandes custos para a empresa por se tratar de uma expansão do sistema atual, tornando-se inviável, se considerarmos a frequência na qual ele será utilizado, ou seja, apenas uma vez por ano.

\section{RESULTADOS}

Parametrização

A parametrização do sistema desenvolvido deverá ser realizada pela Comissão Eleitoral (C.E.) que receberá treinamento previamente.

Na sequência, é apresentado o Diagrama de Contexto (figura 1), sendo o mesmo capaz de demonstrar o local onde cada informação ficará armazenada no sistema desenvolvido. É evidenciado um nível genérico, quais atividades são responsabilidades do sistema e quais não são.

O diagrama de contexto mostra as entidades externas, fluxo de dados e o processo ou função.

\subsection{Teste Piloto}


Figura 1. Diagrama de contexto

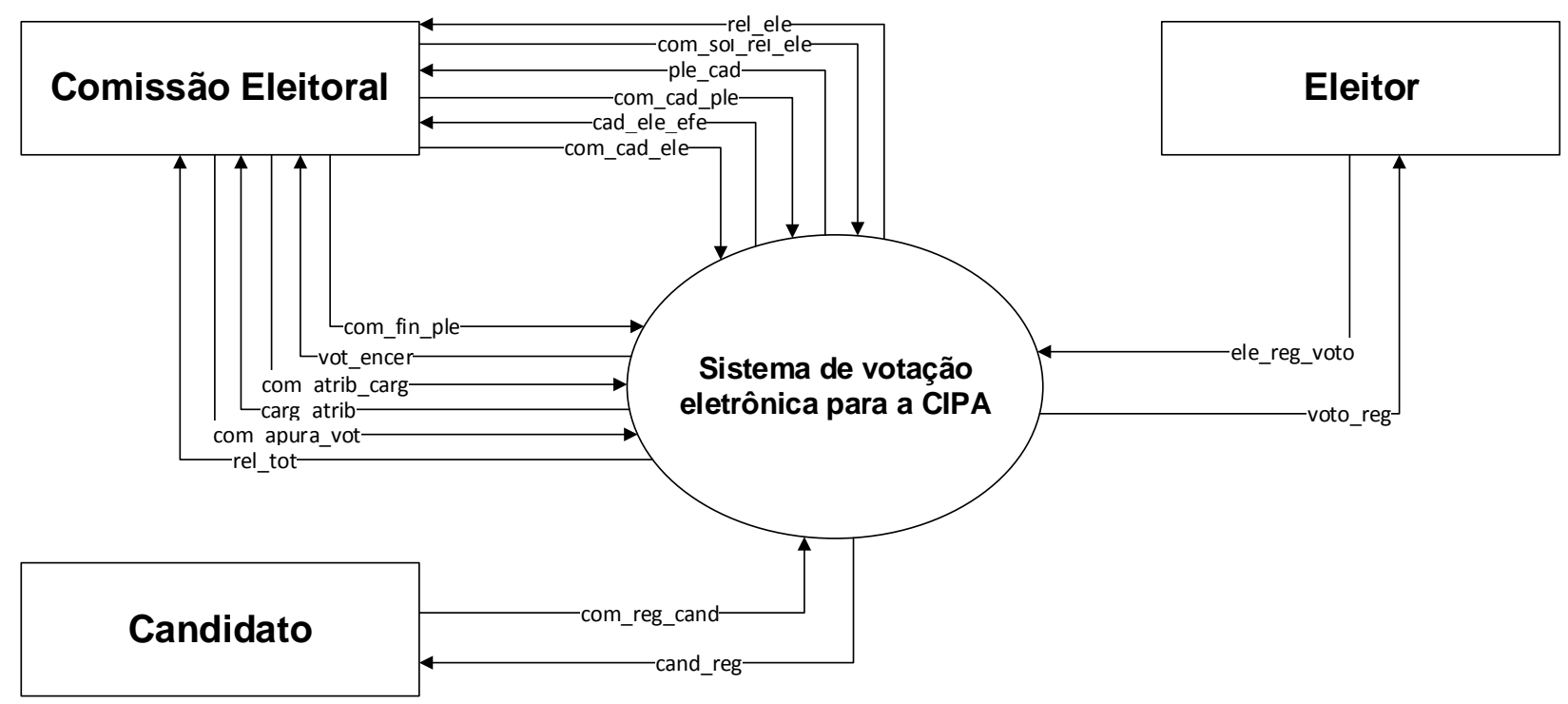

Fonte: (ALVES, 2015).

\subsection{Processo de votação}

No processo de votação, pessoas com diferentes níveis de conhecimento em informática irão manusear o sistema, sob a supervisão da Comissão Eleitoral. Portanto, o sistema disponibilizará uma interface simples e objetiva, o eleitor também poderá contar com a ajuda de um supervisor da Comissão Eleitoral (CE) para realizar seu voto, caso necessário. Ao final da votação, a comissão irá acessar a área restrita para apurar os votos.

Na sequência, é apresentado o Diagrama de Estrutura de Telas (Figura 2). Ou seja, contempla-se a estrutura física das telas, como elas são posicionadas no sistema e suas respectivas ligações com as demais telas.

Figura 2. Diagrama de Estrutura de Tela

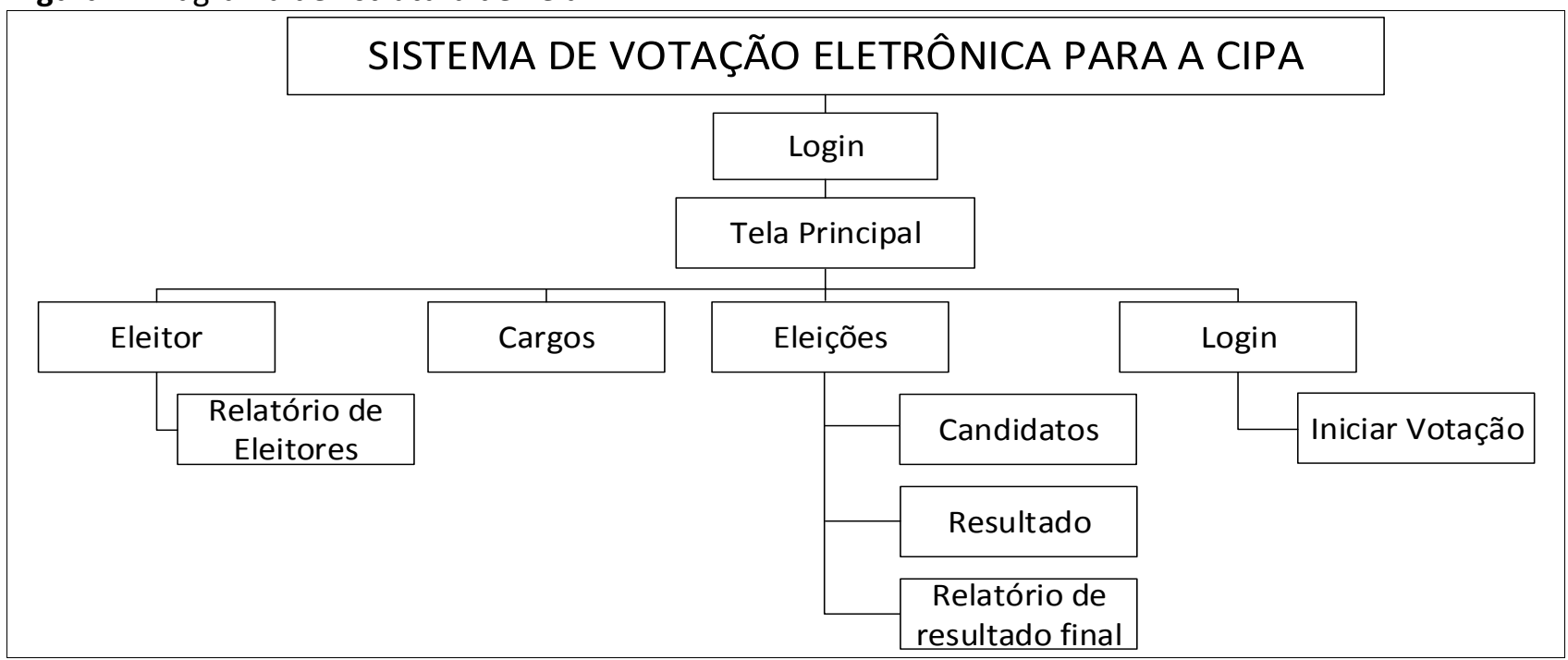

Fonte: (ALVES, 2015).

Na Figura 3, é apresentado o relatório com o resultado das eleições e os devidos cargos atribuídos. 
Figura 3. Relatório de resultado das Eleições

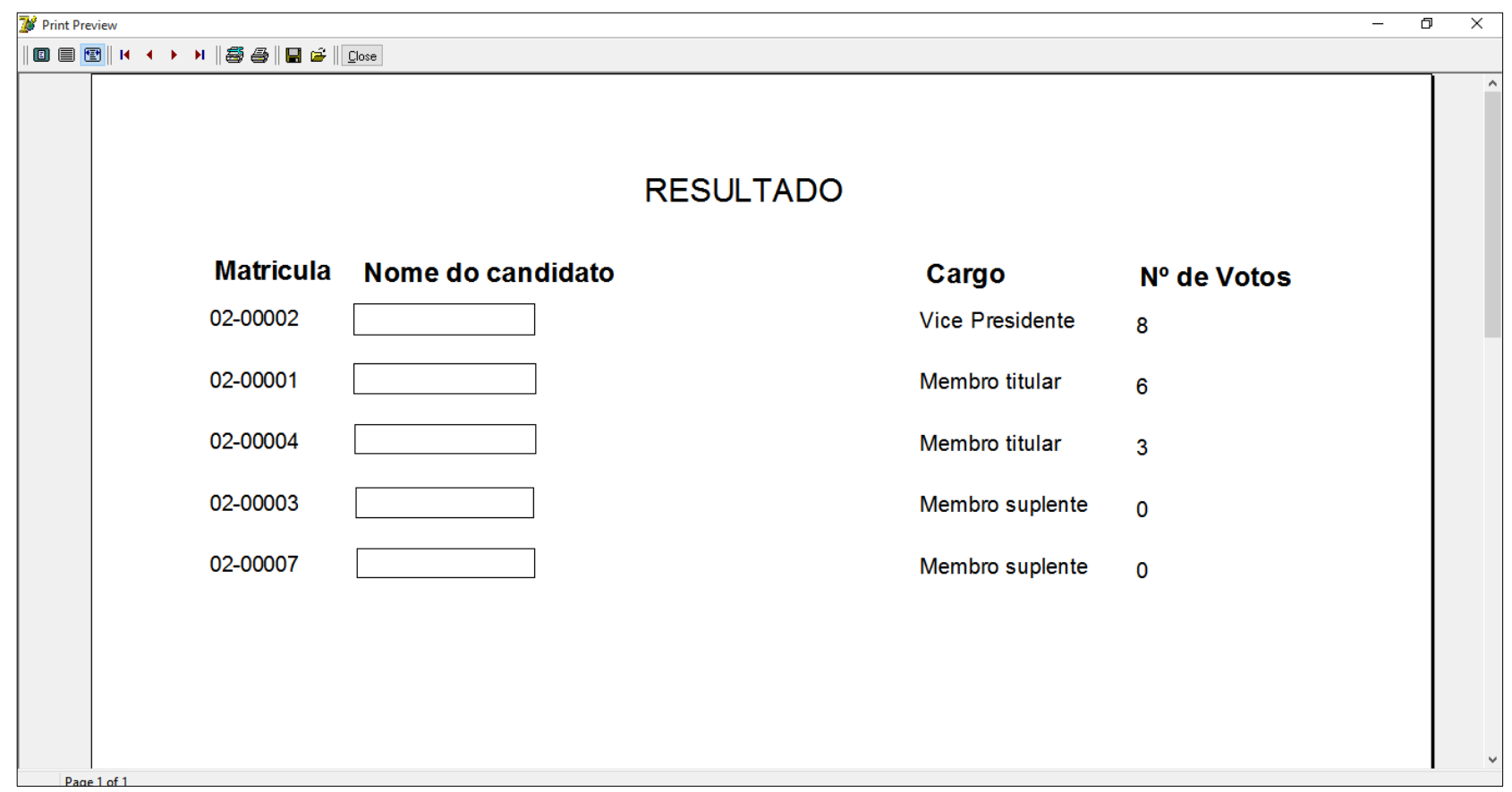

Fonte: (ALVES, 2015).

\section{DISCUSSÃO}

Um programa como o apresentado é capaz de agilizar o processo para se atingir o resultado final de uma eleição. O referido serviu à época para uma empresa que, mantinha mais de cento e trinta funcionários (ALVES, 2015).

Nessa perspectiva, ressalta-se que a tecnologia auxilia na economia de tempo com processos manuais, que aumentam as possibilidades de erros humanos, além de demandarem de disponibilidade dos funcionários envolvidos (TENÓRIO, 2014).

Ainda sobre as possibilidades de agilizar o processo e melhorar o nível de segurança, vale a pena ressaltar que é possível adicionar um leitor biométrico para liberar o candidato a realizar seu voto, ao invés solicitar o número de sua matrícula na empresa. Para tanto, pode-se utilizar a base de dados do relógio de ponto, no qual se encontram todas as informações relacionadas a cada funcionário, incluindo as de suas digitais.

De acordo com Schauren (2016), Farias e Carmo (2017) e Oliveira Junior (2017), o leitor biométrico foi incorporado à urna eletrônica brasileira para tornar mais rígido o processo de identificação do eleitor e o acesso à máquina de votação, a fim de resguardar o sigilo do voto e eliminar a possibilidade de fraudes.

\section{CONSIDERAÇÕES FINAIS}

O desenvolvimento desse sistema trouxe grandes desafios, sendo que foi possível criar uma ferramenta para atuar na área de Segurança do Trabalho, por meio de ferramentas computacionais de baixo custo, ao atender os principais anseios da empresa. Por meio do estudo piloto, na empresa AME - Andradina-SP, constatou-se que com o crescimento da empresa, uma ferramenta adequada para ajudar no processo de votação e apuração dos votos da comissão CIPA é imprescindível, dispondo de baixo custo de investimento, manutenção, além de agilizar todo o processo envolvido.

A tecnologia da informação nos mostra horizontes intangíveis, tornando-se fundamental dentro das empresas. Ela é útil no processo de gerenciamento, na disponibilização de informações imediatas e seguras. Além de agilizar os processos, facilita a comunicação e impacta no lucro e na competitividade das empresas (RIO; MOURA, 2016)

\section{REFERÊNCIAS}

ALVES, R.; Sistema de votação eletrônica para a Cipa. 2015. 45f. Trabalho de Conclusão de Curso (Graduação em Tecnologia em Análise e Desenvolvimento de Sistemas), Fundação Educacional de Andradina - FISMA, Andradina, 2015. 
CÔRTES, P. L. Administração de Sistemas de Informação. São Paulo: Editora Saraiva, 2008.

CRISTOVÃO, L. Object Pascal para Delphi. Florianópolis: Editora Visual Books, 2002.

FARIAS, D. S.; CARMO, S. A. L. M. Técnologias Biométricas: segurança da informação. 2017. $15 f$. Trabalho de Conclusão de Curso (Engenharia), Universidade do Porto, Porto-PT, . 2017.

OLIVEIRA JUNIOR, N. Sistema de eleição seguro o eficiente. 2017. 26f. Trabalho de Conclusão de Curso (Bacharelado em Sistemas de Informação), Universidade Federal de Uberlândia, Uberlândia, 2017.

PACHECO, R. C. S.; TAIT, T. F. C. Tecnologias de Informação: Evolução e Aplicações. Teor. Evid. Econ., Passo Fundo, v. 8, n. 14, p. 97-113, mai. 2000.

RIO, A. P. R.; MOURA, T. M. M. Auxílio da TI no gerenciamento das empresas. 2016, $59 \mathrm{f}$. Trabalho de Conclusão de Curso (Tecnologia em Sistemas de Computação), Universidade Federal Fluminense, Niterói, 2016.

SCHAUREN L. F. Segurança no sistema brasileiro de votação eletrônica. 2016. 40f. Monografia (Bacharelado em Ciências da Computação), Universidade Federal do Rio Grande do Sul Instituto de Informática, Porto Alegre, 2016.

SEGURANÇA E MEDICINA DO TRABALHO. Lei no 6.514, de 22 de Dezembro de 1977; Normas Regulamentadoras - NR, aprovadas pela Portaria no 3.214, de 8 de junho de 1978; Índices Remissivos. 60. ed. São Paulo: Editora Atlas, 2007

TENÓRIO, F. G. Tecnologia da Informação transformando as organizações e o trabalho. Rio de Janeiro: Editora FGV, 2014. 